\title{
Universal temperature dependence of the magnetization of gapped spin chains
}

\author{
Yoshitaka Maeda \\ The University of British Columbia, Vancouver, British Columbia, Canada V6T 1 Z1 \\ Chisa Hotta \\ Aoyama Gakuin University, 229-8558, Sagamihara, Japan \\ Masaki Oshikawa \\ Institute of Solid State Physics, University of Tokyo, Kashiwa 277-8581, Japan
}

\begin{abstract}
Temperature dependence of the magnetization of the Haldane spin chain at finite magnetic field is analyzed systematically. Quantum Monte Carlo data indicates a clear minimum of magnetization as a function of temperature in the gapless regime. On the basis of the Tomonaga-Luttinger liquid theory, we argue that this minimum is rather universal and can be observed for general axially symmetric quasi-one-dimensional spin systems. Our argument is confirmed by the magnetic-field dependence of the spin-wave velocity obtained numerically. One can estimate a magnitude of the gap of any such systems by fitting the experimental data with the magnetization minimum.
\end{abstract}

PACS numbers: $75.10 . J m$

\section{INTRODUCTION}

The discovery of the Haldane gap $\underline{\underline{1}}$ established an important paradigm followed by the extensive studies on gapped spin chains. In particular, recent remarkable progress in high magnetic field experiments provide various data on the closing of the gap by the applied magnetic field. They stimulate a renewed interest in corresponding theoretical studies. However, because of methodological reasons the theories on gapped spin systems still lie far behind those of the exactly solvable $S=1 / 2$ Heisenberg chain ${ }^{2}$. Actually at present the only clue to understand the experiments are the phenomenological effective theories ${ }^{3.4}$ and the numerical calculations which support them.

In this paper, we study the temperature dependence of the magnetization of the $S=1$ Haldane chain for wide range of magnetic field. We particularly focus on the cases where the magnetic field, $h$, exceeds the gap, $\Delta$. The magnetization is one of the most fundamental quantity observed in experiments. In spite of the simplicity of the problem, it is not easy to give a systematic understanding of the magnetization in such systems. Numerical approaches to the problem also had difficulties. For example, quantum Monte Carlo (QMC) method $5,6,7$ had difficulty due to small acceptance ratio in a finite magnetic field. This was overcome very recently $\underline{8,9,10,11,12}$ with new formulations such as Stochastic Series Expansion (SSE). Taking advantage of such developments, we revisit the problem by combining various analytical approaches with numerical results obtained with modern techniques (QMC with SSE, and density matrix renormalization group(DMRG)). We clarify universal features in the temperature dependence of the magnetization in the Haldane chain under an applied magnetic field. Our findings are applicable to general gapped one-dimensional spin systems, as well.

The Hamiltonian of the Heisenberg spin chain is given by

$$
\mathcal{H}=J \sum_{j=1}^{N} \boldsymbol{S}_{j} \cdot \boldsymbol{S}_{j+1}-h \sum_{j=1}^{N} S_{j}^{z},
$$

where $J>0$ and $N$ is the system size. The ground state of $S=1$ Haldane chain is non-magnetic (a singlet state) and has a finite energy gap, $\Delta=0.4105 \mathrm{~J}$, which is estimated numerically $\underline{13.14}$. The system undergoes a quantum phase transition at finite magnetic field, $h=h_{c}(=\Delta)$; in the Haldane gap phase $h<h_{c}$, the magnetization exponentially vanishes toward zero temperature, which is confirmed experimentally, e.g. in Ref. 15. On the other hand, in the $h \geq h_{c}$ phase (gapless regime) the magnetization is finite even at $T=0$. This regime can be described by the TomonagaLuttinger (TL) liquid ${ }^{3}$ as in the $S=1 / 2$ Heisenberg chain. Therefore, one might expect that its magnetization also follows that of the $S=1 / 2$ Heisenberg chain, which is well-known as the Bonner-Fisher curve $\underline{16}$. This is, however, not the case. We show that the magnetization curve as a function of $T$ in the $S=1$ gapless regime has a minimum at $T=T_{m}$ and that the value of $T_{m}$ depends on the magnetic field. Although this behavior resembles that of the three-dimensional Bose-Einstein condensation (BEC) of magnons ${ }^{17}$, the origin obviously differs between the two cases since the BEC at $T \neq 0$ never occur in the one-dimensional systems $\frac{18}{2}$. We prove that this phenomena can be observed in general one-dimensional spin systems (chains and ladders) with the rotational symmetry about the magnetic field direction (axial symmetry). 
The magnetization of the $S=1$ gapless phase has already been studied in many articles; the field dependence of the magnetization is presented at the several fixed values of temperature $19,20,21$, while the particular details of its temperature dependence is overlooked. Hida et al. discussed in the sine-Gordon model ${ }^{22}$ the winding numbers induced by the chemical potentials, which can be interpreted as the magnetization due to the finite magnetic field in the Haldane chain. This mapping describes correctly the $h \sim h_{c}$ case. However, it breaks down at higher magnetic fields. Konik et al. .23 $^{2}$ derived the susceptibility $\chi(h)$ as a function of $h$ on the basis of the exact solution of the nonlinear sigma (NL $\sigma$ ) model (see $M=\int \chi(h) d h$ in Fig.5 of Ref 23). Unfortunately, at finite magnetization, the NL $\sigma$ model does not give accurate descriptions as we will discuss shortly. In this way, the comprehensive understanding of magnetization in the entire gapless regime $h_{c}<h<h_{s}$ was lacking, which we clarify in the present paper. Various universal features are found which also apply to other one-dimensional gapped spin systems.

We present our findings in the following manner; In Sec. II we give the QMC results for the magnetization of the $S=1$ Haldane chain. In Sec. III, we elucidate the universal features of the temperature dependence of the magnetization, and explain the numerical results. Our findings on purely one-dimensional systems are also compared with the magnon Bose-Einstein condensation in three dimensions.

\section{NUMERICAL RESULTS}

Let us first remind of the temperature dependence of the magnetization of the $S=1 / 2$ Heisenberg chain as a reference. At present precise numerical data are easily obtained on the basis of the Bethe Ansatz solution (using the integrability of the Quantum Transfer Matrix ${ }^{24}$ ) as shown in fig. 1 for the various magnetic fields. For any value of the magnetic field, as a function of the temperature, the magnetization monotonically increases until it hits a maximum, and then monotonically decreases down to zero in the infinite temperature limit. This behavior is qualitatively the same as in the classical spin resultes. The XXZ chain in the gapless regime also shows a similar behavior. The decrease at low temperature is intuitively interpreted as the growth of the antiferromagnetic short range order.

Next, we present the QMC results for the $S=1$ Haldane chain. The QMC simulation is performed at $N=512$ with the maximum 1200000 Monte-Carlo step using a stochastic series expansion $10,11,12$ code of the ALPS library $26,27,28$. The convergence in the thermodynamic limit after the finite size scaling is confirmed by the comparison of our results with the high temperature expansion results. Figures 2 shows the magnetization in the Haldane gap phase $h<h_{c}$ of eq. (11). As well known, it monotonically decreases and vanishes toward $T \rightarrow 0$ following $T^{-1} \exp (-\Delta / T)$. In contrast, the magnetization at $h>h_{c}$ has a characteristic structure. Figures 3(a) and 3(b) show the magnetizations at $h_{c}<h<h_{s}=4 J\left(h_{s}\right.$ is the saturation field). As in the $S=1 / 2$ case, the qualitative behavior is consistent with that of the classical spin system except at low temperature. However, unlike the $S=1 / 2$ case the magnetization minimum is observed at $T=T_{m}$. The temperature $T_{m}$, which gives the magnetization minimum, decreases toward $T=0$ as $h$ is lowered to $h_{c}$.

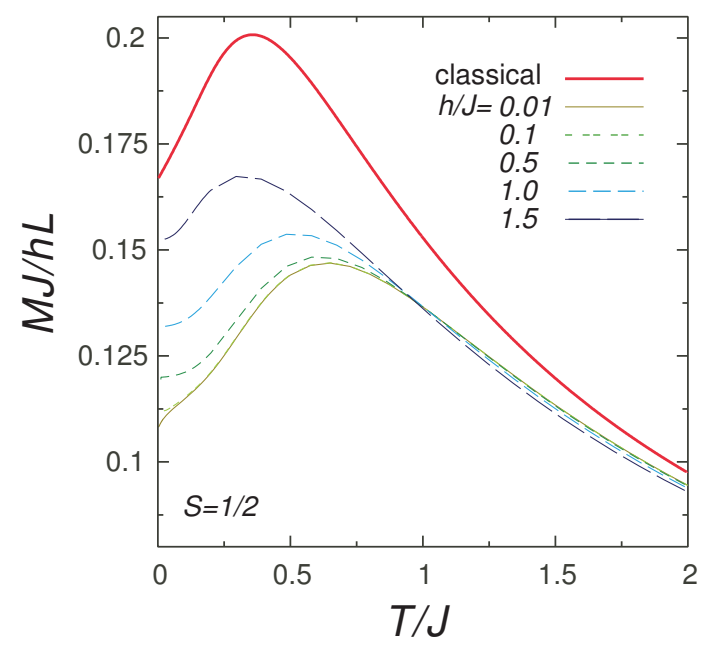

FIG. 1: The temperature dependence of the magnetizations of the $S=1 / 2$ Heisenberg model. The exact classical spin solution and the Bethe ansatz results for several choices of $(h / J=0.01 \sim 1.5)$ are depicted. 


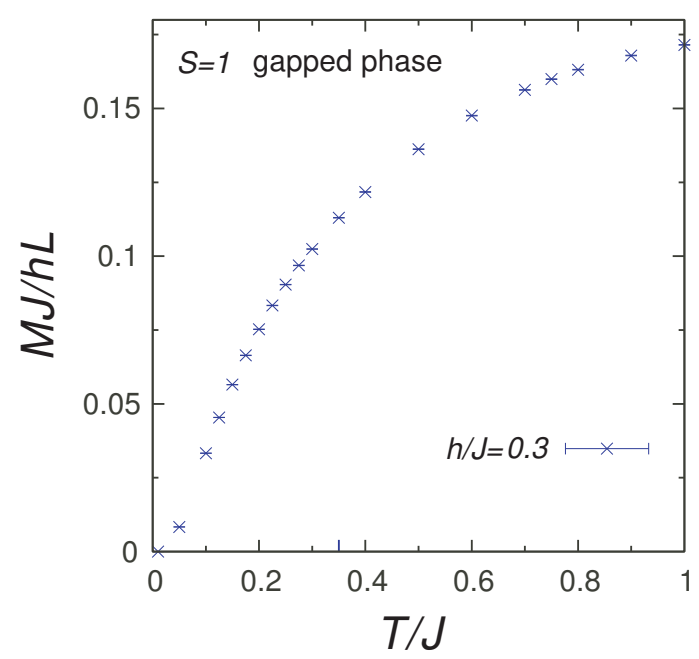

FIG. 2: The temperature dependence of the magnetization of the $S=1$ Heisenberg model for $h\left(<h_{c}\right): h=0.3 J$. In this regime, the temperature dependence monotonically decreases; $T^{-1} \exp (-\Delta / T)$, especially for $T \ll \Delta$. The error bar of each point is much smaller than the symbol size.
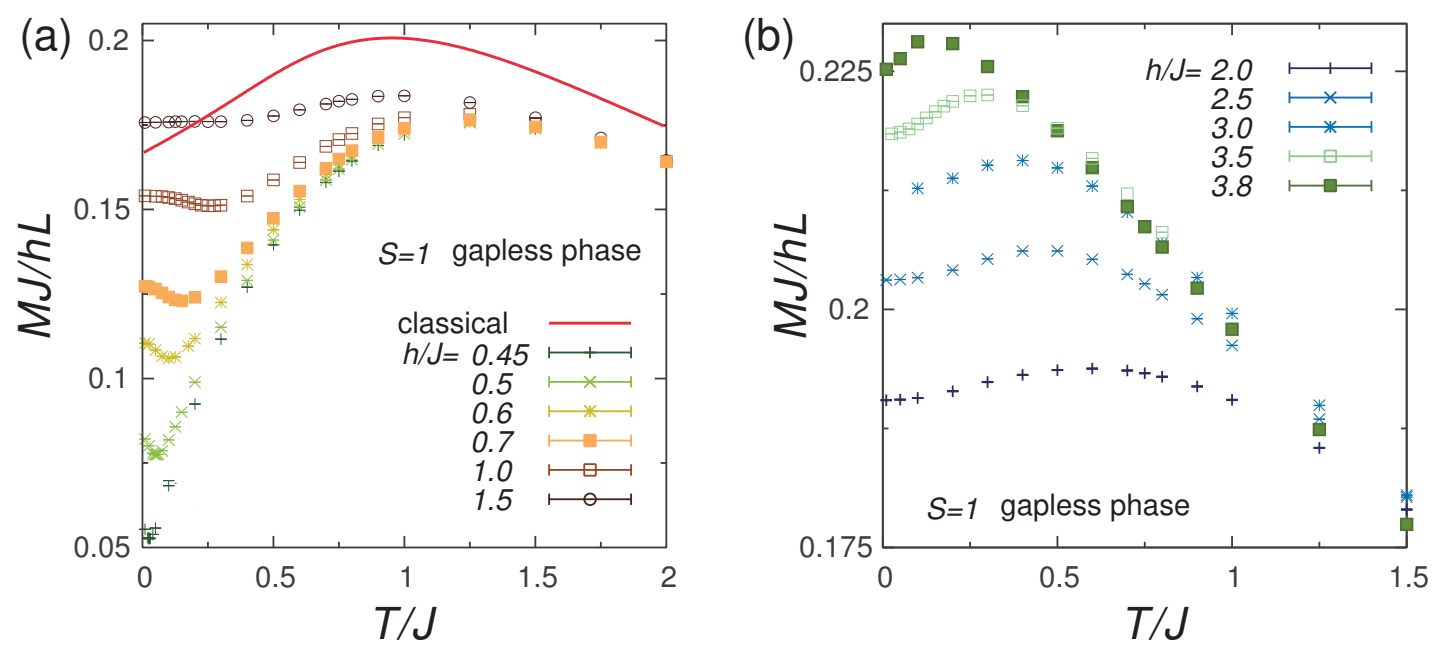

FIG. 3: The temperature dependence of the magnetization of the $S=1$ Heisenberg model in the gapless phase $\left(h>h_{c}\right)$ below the saturation field $\left(h_{s}=4.0 J\right)$ at several values of $h / J$, (a)0.45-1.5, and (b)2.0-3.8. The exact susceptibility of the classical spin Hamiltonian is shown together in (a). The error bar of each point is much smaller than the symbol size.

\section{DISCUSSION}

We discuss the characteristic behavior found in Fig. 3 by use of generic effective theories of the gapped spin chains with short-range interactions. Note that the logic given in this section are not restricted to the Haldane chain.

\section{A. Effective theory near $h_{c}$ and $h_{s}$}

Excited states above the gap from the $S^{z}=0$ ground state generally consists of a triplet massive boson state with $S^{z}= \pm 1,0$ which usually have repulsive short-range interactions. Low-energy states of these particles may be approximated by a non-relativistic dispersion relation:

$$
E(k) \approx \Delta+k^{2} / 2 m-h S^{z}
$$


where $m$ is a band curvature. If an underlying relativistic theory exists, which is not necessarily the case, $v_{0}=\sqrt{\Delta / m}$ corresponds to the relativistic "speed of light". As a result, the $S^{z}=1$ magnon branch intersect with the ground state at $h=h_{c}=\Delta$, a quantum phase transition point. In the gapless regime a quasi long range order appears ${ }^{3}$, where one may integrate out the $S^{z}=0,-1$ magnons which are the higher energy states. Then, in the low density limit of the magnons, $h=h_{c}$, the remaining $S^{z}=1$ magnons can be exactly mapped onto the free fermion theory with the dispersion eq. (2) $3,29,30$. The low but finite magnon density regime, $h \gtrsim h_{c}$, can be still approximately described by the free fermion theory since residual interactions are proportional to $h-h_{c}$. Here, the magnetization is equal to the number of the particle as

$$
\frac{M}{L}=\sqrt{\frac{m}{2 \pi^{2}}} \int_{0}^{\infty} d \epsilon D(\epsilon) f(\epsilon-\mu),
$$

where $\epsilon=k^{2} / 2 m, \mu=h-\Delta$, and $f(\epsilon-\mu)=1 /\{\exp (\beta(\epsilon-\mu))+1\}$ is the Fermi distribution function. The density of states follow, $D(\epsilon) \propto 1 / \sqrt{\epsilon}$, constant, and $\sqrt{\epsilon}$ in one, two, and three-dimensions, respectively. Then, from eq. (3), the increase of the magnetization at $T \rightarrow 0$ in Fig 3 can be easily understood by the following argument; the Fermi distribution function is symmetric with respect to the Fermi point $\mu$, namely, $f(\epsilon+\delta \epsilon-\mu)=1-f(\epsilon-\delta \epsilon-\mu)$. Moreover, $f(\epsilon-\mu)$ for $\epsilon>2 \mu$ near $T=0$ is negligibly small, since $f(\epsilon-\mu) \sim \exp (-\beta(\epsilon-\mu))$. Therefore, if the density of states increases for lower energy $\epsilon$, so does eq. (3) at $T \rightarrow 0$. From this observation, we can conclude that this decrease of the magnetization in fig. 3 stems from the singularity of the density of states in one-dimensional systems. The exact integration of eq. (3) gives the explicit expression,

$$
\frac{M}{L}=-\sqrt{\frac{m}{2 \pi \beta}} \operatorname{Li}_{n=1 / 2}\left[-e^{\beta(h-\Delta)}\right],
$$

where $\operatorname{Li}_{n}[x]=\sum_{l=1}^{\infty} x^{l} / l^{n}$ is the polylogarithm function. Actually, the numerical description of eq. (44) in fig. (4 reproduces well the minimum of $M$ found in fig. 3(a).

The analogous mapping between the dilute boson and the free fermion holds at $h \lesssim h_{s}$ as well. In this case, the vacuum state is the fully-polarized state and the low-lying excitations consist of $S^{z}=0$ magnon branch instead of the $S^{z}=1$ one. Then, the magnetization shows a maximum at low temperature corresponding to the minimum of the magnon density, just the opposite to that at $h \gtrsim h_{c}$. The behavior found in fig. 3 (b) (e.g $\left.h=3.5,3.8\right)$ are consistent with this argument.

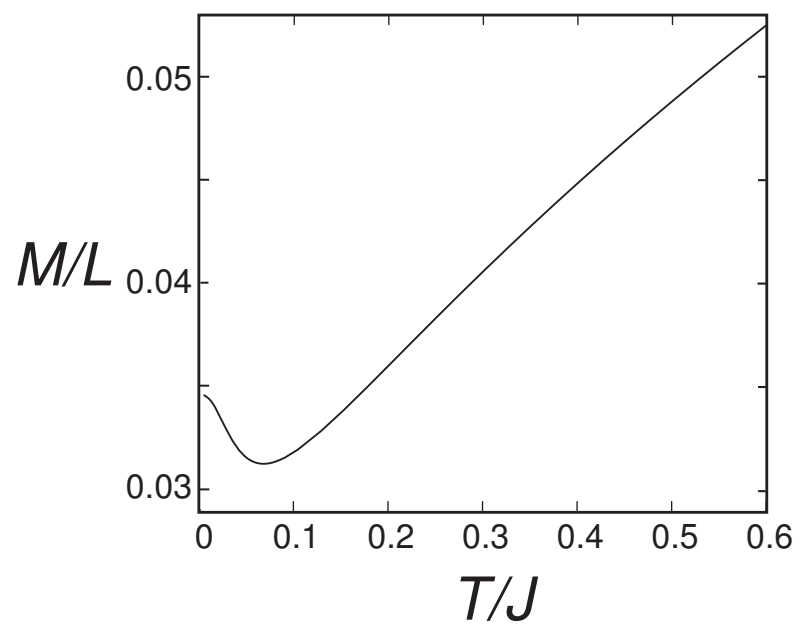

FIG. 4: The free fermion result, eq. (4) for $h-\Delta=0.3$, is depicted.

B. Effective theory in the low-energy limit for $h_{c} \leq h \leq h_{s}$

The free fermion description has thus succeeded in reproducing the minimum/maximum in fig. 3. However, it is valid only slightly above $h_{c}$ and below $h_{s}$. On the other hand, the TL liquid theory should be applicable for the whole gapless regime in one-dimensional systems $\left(h_{c}<h<h_{s}\right.$ in the present case), albeit only in the low energy limit. In 
the following, we apply the TL liquid theory to estimate the magnetization. From the conformal field theory, the low temperature expansion of the free energy per unit volume is given by ${ }^{31,32}$

$$
f=\epsilon_{0}-\frac{\pi c}{6 v_{F}} T^{2}+O\left(T^{3}\right)
$$

where $\epsilon_{0}$ is an ground state energy, $v_{F}$ is the excitation velocity of a fixed point theory, and $c$ is the central charge $(c=1$ for TL liquid). The magnetization is given by the derivative of the free energy with respect to the magnetic field,

$$
\begin{aligned}
\frac{M}{L} & =-\partial_{h} f \\
& =\frac{M_{0}}{L}-\frac{\pi}{6 v_{F}^{2}} \frac{\partial v_{F}}{\partial h} T^{2}+O\left(T^{3}\right) .
\end{aligned}
$$

The first and the second term give the magnetization of the ground state and the leading finite temperature correction, respectively. This equation indicates that whether the magnetization near $T=0$ increases or decreases is determined by the sign of the gradient of the velocity with respect to the magnetic field, $\partial_{h} v_{F}$. For the $S=1 / 2$ Heisenberg chain, we always have $\partial_{h} v<0$ (see fig. 9 in ref. 34), which is consistent with Fig. 1. In contrast, the gapless TL liquid regime in the present case has $v_{F}=0$ at both endpoints, $h=h_{c}$ and $h=h_{s}$, so that $v_{F}$ must have a maximum in between. This indeed gives the characteristic behavior observed in Fig. 3 ,

Let us demonstrate the validity of eq. (6) in the special case of $h \gtrsim h_{c}$, where an explicit evaluation of $v_{F}(h)$ is available. Following the free-fermion dispersion in eq. (2) we have

$$
v_{F}=v_{0} \sqrt{\frac{2(h-\Delta)}{\Delta}}
$$

The low temperature expansion of the magnetization given by the free-fermion theory is derived from the Sommerfeld expansion $^{35}$ on eq. (3) as,

$$
\frac{M}{L}=\frac{\sqrt{2 \Delta(h-\Delta)}}{\pi v_{0}}-\frac{\pi}{12 v_{0}} \sqrt{\frac{\Delta}{2}}(h-\Delta)^{-3 / 2} T^{2}+O\left(T^{4}\right),
$$

with $v_{0}^{2}=\Delta / m$. These two equations lead to

$$
\frac{M}{L}=\frac{\sqrt{2 \Delta(h-\Delta)}}{\pi v_{0}}-\frac{\pi}{6 v_{F}^{2}} \frac{d v_{F}}{d h} T^{2}+O\left(T^{4}\right)
$$

which is the special case $h \gtrsim h_{c}$ of the general result, eq. (6). The behavior of $M / L$ at $h \lesssim h_{s}$ is analogously obtained by replacing $v_{F}$ in eq.(7) with $v_{F} \propto \sqrt{h_{s}-h}$, following the mapping of $S^{z}=1$ at $h \gtrsim h_{c}$ magnons with $S^{z}=0$ magnons at $h \lesssim h_{s}$.

To confirm the above argument we obtain $v_{F}$, by the DMRG calculation on the $S=1$ Haldane chain with periodic boundary condition. The energy at the fixed magnetization, $M / L$, is obtained for discrete values of $M=0-L$ with system size up to $L=120$. One can fit the energy values, $E(M / L, L)$, obtained after the extrapolation of truncation error to the finite size scaling:

$$
\frac{E(M / L, L)}{L}=\epsilon_{o}(M / L)-\frac{\pi v_{F}(M / L)}{6} \frac{1}{L^{2}}+O\left(1 / L^{3}\right),
$$

where $\epsilon_{o}(M / L)$ and $v_{F}(M / L)$ denote the energy and velocity at finite magnetization, respectively. Figure[5(a) shows the magnetization curve which is obtained from the energy as a function of $M / L$ in its the inset. By use of this $M / L-h$ curve, we plot in Fig. 5 (b) the velocity as a function of the magnetic field. The velocity increases rapidly just above $h=h_{c}$, takes a maximum at around $h \sim J$, and then decreases toward $h=h_{s}$, as anticipated in the previous discussion. The reason why the gradient of $v_{F}$ shows a sudden change at $h \sim J$ and $3 J$ is still out of our scheme, while the shape of $v_{F}$ in the whole gapless regime suggests that it might be divided into three parts according to the change of nature in its excitation spectrum. Going back to Fig. 3 we find several cases where the $M-T$ curves are almost flat in a fairly wide temperature range, e.g., $h / J=1.5,2.0$. Apparently, this is consistent with the fact that the velocity $v_{F}$ takes the maximum $\left(\partial_{h} v_{F}=0\right)$ at a certain field within this range, as shown in Fig. 5 (b). At this field, the leading finite temperature correction starts from $O\left(T^{3}\right)$. Even when $h$ is slightly shifted from the maximum, $v_{F}$ depends rather weakly on $h$. Thus for the range of $h$ around the maximum, magnetization looks almost flat in the low temperature regime. 

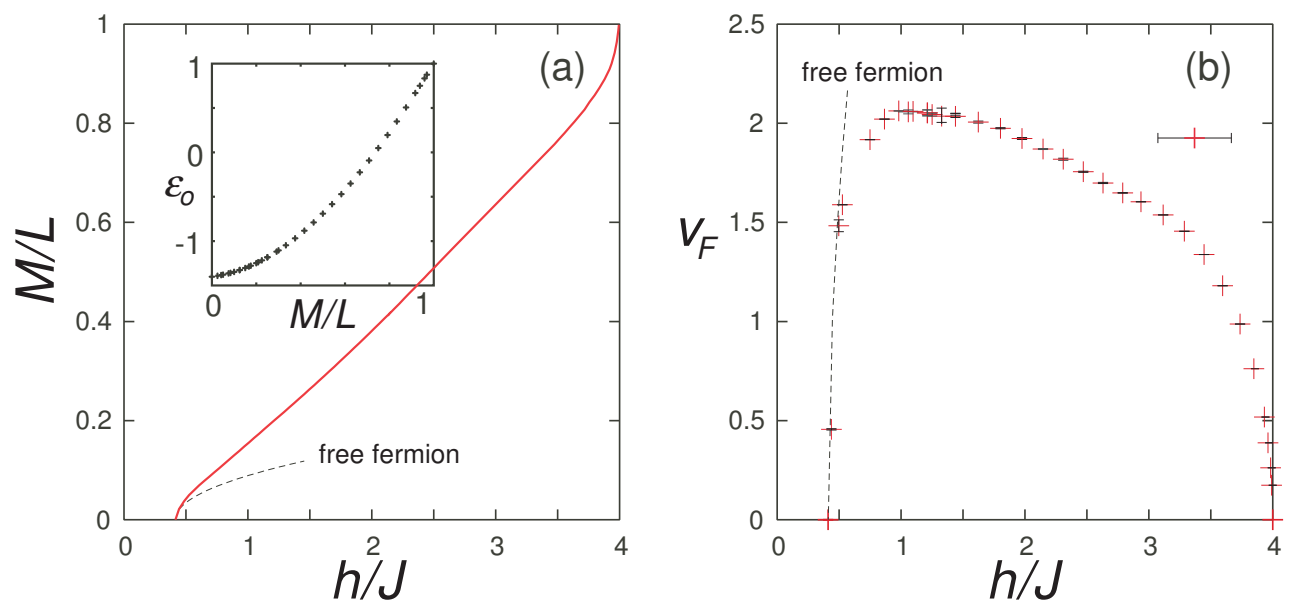

FIG. 5: (a) Magnetization as a function of magnetic field of the $S=1$ Haldane chain in the bulk limit. The inset shows the $M / L$ dependence of energy per site obtained by DMRG with periodic boundary condition up to $L=120$. The energy at each $L$ is extrapolated against the truncation error, where the accuracy is guaranteed to less than the order of $10^{-6}$ in the worst case. (b)The magnetic field dependence of velocity derived from the data in (a). The broken lines in (a) and (b) are the free fermion values taken from the first term of eq.(9) and from eq.(7) with $v_{0} / J=2.49^{33}$, respectively.

So far, we have not discussed the effects of irrelevant operators on eq. (5). Generally, Lorentz invariant terms never renormalize the spin-wave velocity which corresponds to the "speed of light". Therefore, to examine whether the corrections due to irrelevant operators are required in eq. (6), perturbations which break Lorentz invariance should be explicitly considered. In principle, irrelevant perturbations could give larger corrections than $T^{2}$, e.g. in the case of the $S=1 / 2$ Heisenberg chain, the marginally irrelevant operator gives a logarithmic correction to the zero magnetic field susceptibility $\underline{36}$. Generally, under the compactification of the bosonic field $\phi$, the vertex operator $\exp (i \alpha \phi)(\alpha$ is a constant parameter) and $\left(\partial_{x}^{m} \phi\right)^{n}(\mathrm{~m}$ and $\mathrm{n}$ are integers) can be perturbations around the TL liquid fixed point. In the present case with $M \neq 0$, the former one became less relevant in the low temperature regime, $T \ll M / J$. This is because it usually takes a form of $\exp (i M x / L) \times \exp (\alpha \phi)$, remaining negligible in the low energy limit. Then, the $\left(\partial_{x} \phi\right)^{3}$-term becomes the leading perturbation, since the $\partial_{x} \phi$ and $\left(\partial_{x} \phi\right)^{2}$-terms are exactly absorbed into $\mathcal{L}_{0}$ and $\partial_{x}^{2} \phi$ is forbidden by the parity symmetry $(x \rightarrow-x$ and $\phi \rightarrow-\phi)$. By a standard dimension analysis, this term again leads to a $T^{2}$ correction to eq. (6). This correction should be understood as already included in eq. (6), which thus stands valid.

\section{Crossover temperature and estimation of the gap}

Let us now return to the discussion to the characteristic temperature that give the magnetization minimum, $T_{m}$. It can be interpreted as a crossover temperature from the TL liquid to the state with non-relativistic dispersion, $\epsilon \propto k^{2}$, which is indicated by the arrow in fig. 6. At $T \ll h-h_{c}$, the system can be described by a TL liquid with linearized dispersions around the two Fermi points $\left(\propto \sqrt{h-h_{c}}\right)$. However, with increasing $T \gtrsim h-\Delta$, the effect of band curvature becomes more important due to thermal excitations.

Here we propose a new way to estimate a gap from $T_{m}$. Equation (44) takes the minimum under the condition, $2 x=\mathrm{Li}_{n=1 / 2}\left[-\mathrm{e}^{x}\right] / \mathrm{Li}_{n=-1 / 2}\left[-\mathrm{e}^{x}\right]$, where $x=\mu / T_{m}$. Its solution, $x=x_{0} \sim 0.76238$, yields

$$
T_{m}=x_{0}(h-\Delta) .
$$

The finite temperature measurement of magnetization at several $h>h_{c}$ in both experiments and numerical calculations thus provide a reasonable estimation of $\Delta$. Since eq. (11) consists only of universal constant we can use it without any microscopic informations of the system. As a demonstration, we show in Fig. 7 the comparison of our Monte carlo results with eq. (11). The data asymptotically approaches eq. (11) toward $h \rightarrow \Delta$ since the deviation at large $h$ is due to the interaction effect of the magnons. Note that all the data at $h>h_{c}=\Delta$ in fig. 7 are located below the values given by eq. (11). This behavior is rather universal for generic models as follows; the excitations to the higher energy modes are enhanced by the repulsive interactions, so that, even in low temperature regime, the band curvature effect in the exact results becomes more important than that in the free case. Consequently, the exact value of the 
crossover temperature, $T_{m}$, must be suppressed by the repulsive interaction compared to the estimate (11) from the free fermion theory.

\section{Comments on previous studies}

In light of our results, we comment on the analysis based on the integrability of the NL $\sigma$ model, as proposed in Ref. 23. They conclude that the spin-wave velocity monotonically increases with the increasing magnetic field, which is contradictory with the results in the present paper. The reason for this discrepancy is that they introduced the finite magnetic field after taking the low-energy limit of the zero-field model, which generally does not give a correct effective theory above the critical field. To illustrate this point, for a moment, let us consider the $S=1 / 2$ Heisenberg antiferromagnetic chain as a simple example. The zero-field effective theory in the low-energy limit is given by the TL liquid, namely the free boson field theory with the Lagrangian density, $\mathcal{L}_{0}=\frac{1}{2}\left(\partial_{\mu} \phi\right)^{2}$, where $\phi$ must be periodic, $\phi \sim \phi+2 \pi R$; $\mathrm{R}$ is the compactification radius 37 . The application of the magnetic field after taking the the low-energy limit can be represented by the term, $\mathcal{L}_{Z}=-\frac{h}{\sqrt{2 \pi}} \partial_{x} \phi$. This formula appears to be easily handled by "completing the square" as $\mathcal{L}_{0}+\mathcal{L}_{Z}=\frac{1}{2}\left(\partial_{\mu} \phi^{\prime}\right)^{2}-\frac{h^{2}}{4 \pi}$, where $\phi^{\prime}=\phi-h x / \sqrt{2 \pi}$. Apparently, there is no renormalization of the spin-wave velocity nor of $R$, which is in clear contradiction to the exact Bethe Ansatz solution 2 . Such fallacy is attributed to taking the low-energy limit first at zero-field, as discussed above. Thus one should note that although the irrelevant perturbations on the fixed point theory $\mathcal{L}_{0}$ can be usually ignored in the low-energy limit, it cannot be in deriving the effective theory at a finite field.

The magnetization minimum has been often discussed in terms of BEC. Although the three-dimensional BEC observed in Ref. 17 gives similar $M-T$ curves to Fig. 3 , it differs from the present case in several points; the magnetization shows a singular cusp of minimum at the transition temperature, $T_{c} \propto\left(h-h_{c}\right)^{2 / 3}$, (which is however smeared in a actual systems ${ }^{38}$ ), whereas $T_{m} \propto h-h_{c}$ in our model just marks the crossover. The transverse magnetization is finite, namely the off-diagonal long-range order is present in BEC at $T<T_{c}$, but is absent in our one-dimensional system at any temperature.

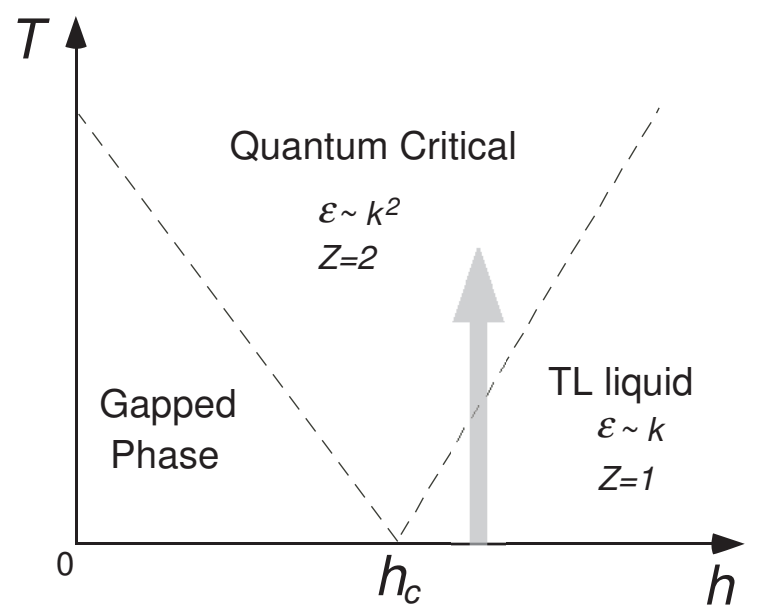

FIG. 6: The phase diagram for the gapped spin chains. Dashed lines indicate the crossovers. The crossover temperature between the TLL regime and the quantum critical regime is $T \sim h-h_{c}$.

\section{SUMMARY}

We found the characteristic temperature dependence of magnetization of the $S=1$ Haldane chain in the gapless regime at finite magnetic field, $h_{c} \leq h \leq h_{s}$. In the vicinity of the endpoints of the gapless regime, $h \gtrsim h_{c}$ and $h \lesssim h_{s}$, the magnetization shows a minimum and maximum, respectively, at the crossover temperature, $T=T_{m}$. The TL liquid theory yields the leading term of magnetization in the low temperature limit as, $M(T, h)-M(T=0, h) \propto \frac{\partial v_{F}(h)}{\partial h} T^{2}$. We showed that the spin wave velocity, $v_{F}(h)$, vanishes at both $h=h_{c}$ and $h_{s}$ and shows a maximum in between. 


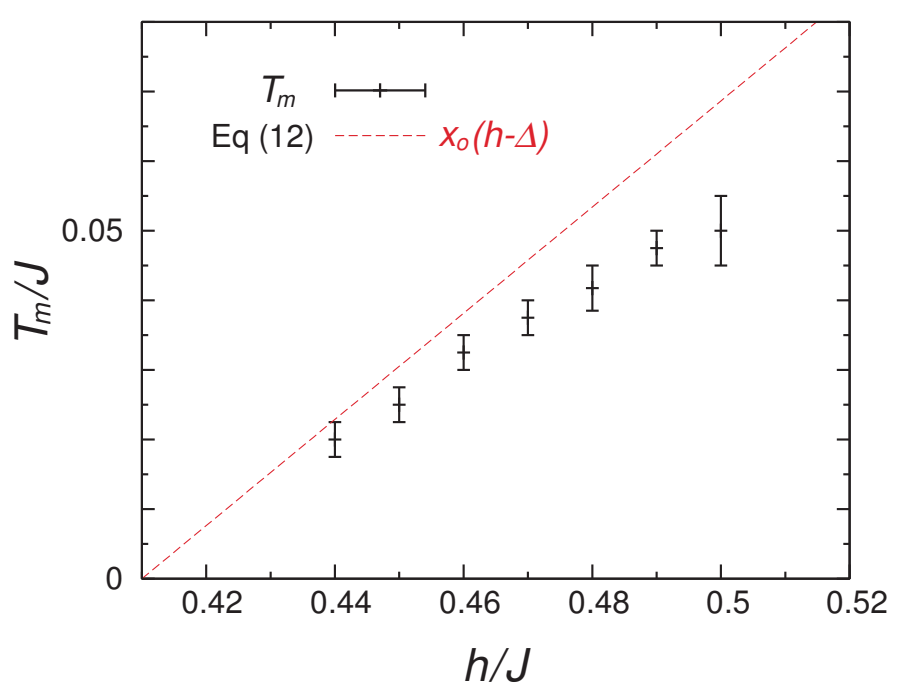

FIG. 7: The fitting of the QMC results with eq. (11). From this fitting, the gap $\Delta$ can be estimated. The error-bars come from a discreteness of the numerical data with respect to temperature.

Therefore, $M(T, h=$ fixed $)$ at around $T \sim 0\left(T \leq T_{m}\right)$ undergoes a change from the decreasing function to the increasing function of $T$ as the magnetic field increases from $h=h_{c}$ to $h_{s}$.

We emphasize that the present discussions are universal (at least) for axial symmetric generic gapped onedimensional spin systems, provided that the field-induced gapless phase can be described by a single-component TL liquid. The detailed structure of $M$ and $v_{F}$ reflecting the characteristics of each system does not violate this scheme since we have dealt with only the universal features in the low-energy limit. Actually, the conclusions do not depend on a particular choice of the effective theory, such as the sine-Gordon model, the nonlinear sigma model, or the delta-function Bose gas.

We also proposed a simple and reliable scheme (represented by eq. (11)) to estimate the gap, $\Delta$, from the finite temperature magnetization measurement.

To our knowledge, there is no reported experimental results corresponding to our analysis in the present paper. However, it might be possible that indeed there are already some corresponding experiments, which have been interpreted in different ways. Actually, the upturn of magnetization at $T=T_{m}$ found in many experiments had been interpreted as a three-dimensional effect (BEC of magnons). However, we argue that it does occur generally in purely one-dimensional gapped spin systems as well. Therefore, a care should be taken in the interpretation of the magnetization data. To distinguish these two scenarios, one should examine whether or not the transverse magnetization exists below $T_{m}$ as well as the presence/absence of the thermodynamic phase transition. In reality the actual magnetic systems have finite interchain interactions. However, the effects described in the present paper may be observable if the interchain interactions are sufficiently weak. We hope that the present work stimulates further study on one-dimensional magnetism.

\section{Acknowledgments}

We would like to thank I. Affleck, J. Akimitsu, H. Tanaka, C. Yasuda, K. Sakai, K. Hida, and S. Todo for fruitful discussions and contributions. This work is supported by a 21st Century COE Program "Nanometer-Scale Quantum Physics" at Tokyo Tech, a 21st Century COE Program at Aoyama Gakuin University, a Grant-in-Aid for Scientific Research under Grant No. 18540341 from MEXT of Japan, and by NSERC of Canada.

${ }^{1}$ F. D. M. Haldane, Phys. Lett. 93A, 464 (1983). For a review, see I. Affleck, J. Phys. Condens. Matter 1, 3047 (1989).

${ }^{2}$ V. E. Korepin, N. M. Bogoliubov and A. G. Izergin, Quantum Inverse Scattering Method and Correlation Functions, Cambridge University Press (1993).

3 I. Affleck, Phys. Rev. B 41, 6697 (1990); I. Affleck, ibid. 43, 3215 (1990). 
4 A. M. Tsvelik, Phys. Rev. B 42, 10499 (1990).

5 M. Suzuki, Prog. Theor. Phys. 561454 (1976); M. Suzuki, S. Miyashita, and A. Kuroda, ibid. 58, 1377 (1977).

${ }_{6}$ M. Roji and S. Miyashita, J. Phys. Soc. Jpn. 65, 1994 (1996).

7 For a review, e.g., H. G. Evertz, The Loop Algorithm, Numerical Methods for Lattice Many-Body Problems, edited by D. J. Scalapino (Addison Wesley, Reading, MA, 1998).

8 N. V. Prokof'ev, B. V. Svistunov, and I. S. Tupitsyn, Pis'ma Zh. Eksp. Teor. Fiz. 64, 853 (1996) [JETP Lett. 64, 911 (1996)].

9 N. V. Prokof'ev, B. V. Svistunov, and I. S. Tupitsyn, Phys. Lett. A 238, 253 (1998); JETP 87, 318 (1998).

10 A. W. Sandvik and J. Kurkijärvi, Phys. Rev. B 43, 5930 (1900).

11 A. W. Sandvik, J. Phys. A 25,3667 (1992).

12 O. F. Syljuåsen and A. W. Sandvik, Phys. Rev. E 66, 046701 (2002).

13 S. R. White and D. A. Huse, Phys. Rev. B 48, 3844 (1993).

14 S. Todo and K. Kato, Phys. Rev. Lett. 87, 047203 (2001).

15 J. P. Renard, M. Verdaguer, L. P. Regnault, W. A. C. Erkelens, J. Rossat-Migdot, and W. G. Stirling, Europhys. Lett. 3, 945 (1987).

16 J. Bonner and M. E. Fisher, Phys. Rev. 135, A640 (1964).

17 T. Nikuni, M. Oshikawa, A. Oosawa, and H. Tanaka, Phys. Rev. Lett. 84, 5868 (2000).

18 P. C. Hohenberg, Phys. Rev. 158, 383 (1967).

19 K. Okunishi Phys. Rev. B 60, 4043 (1999).

20 J. Lou, S. Qin, T. K. Ng, Z. Su, and I. Affleck, Phys. Rev. B 62,3786 (2000)

21 V. A. Kashurnikov, N. V. Prokof'ev, B. V. Svistunov, and M. Troyer, Phys. Rev. B 59, 1162 (1999).

${ }^{22}$ K. Hida, M. Imada, and M. Ishikawa, J. Phys. C: Solid State Phys., bf 164945 (1983).

23 R. M. Konik and P. Fendley Phys. Rev. B 66, 144416 (2002).

${ }^{24}$ F. H. .L. Essler, H. Frahm,F. Göhmann, A. Klümper and V. E. Korepin: The one-dimensional Hubbard model, (Cambridge University Press, Cambridge, 2004).

25 M. E. Fisher, Amer. J. Phys. 32, 343 (1964).

${ }^{26}$ F. Alet et al., J. Phys. Soc. Jpn. Suppl. 74, 30 (2005); see also http://alps.comp-phys.org/.

${ }^{27}$ F. Alet, S. Wessel, and M. Troyer, Phys. Rev. E 71, 036706 (2005).

28 M. Troyer, B. Ammon, and E. Heeb, Lecture Notes in Computer Science, Vol. 1505, p. 191 (1998).

29 H. J. Schulz, Phys. Rev. B 22, 5274 (1980).

30 E. H. Lieb and W. Liniger, Phys. Rev. 130, 1605 (1963); E.H. Lieb, ibid. 130,1616 (1963).

31 I. Affleck, Phys. Rev. Lett. 56, 746 (1986).

32 J. L. Cardy J. Phys. A 17, L385 (1984), and L957 (1984), and Nucl. Phys. B240,[FS12] 514 (1984).

33 E. S. Sørensen and I. Affleck, Phys. Rev. Lett. 71, 1633 (1993).

34 I. Affleck and M. Oshikawa, Phys. Rev. B 601038 (1999).

35 N. W. Ashcroft and N. D. Mermin, Solid state physics (Brooks Cole, 1976).

36 S. Eggert, I. Affleck, and M. Takahashi, Phys. Rev. Lett. 73, 332 (1994).

37 I. Affleck, in Fields, Strings and Critical Phenomena, Les Houches, Session XLIX, ed. by E. Brézin and J. Zinn-Justin, North-Holland, Amsterdam (1988).

38 J. Sirker, A.Weiße, O.P. Sushkov, Europhys. Lett. 68, 275 (2004). 
(a)

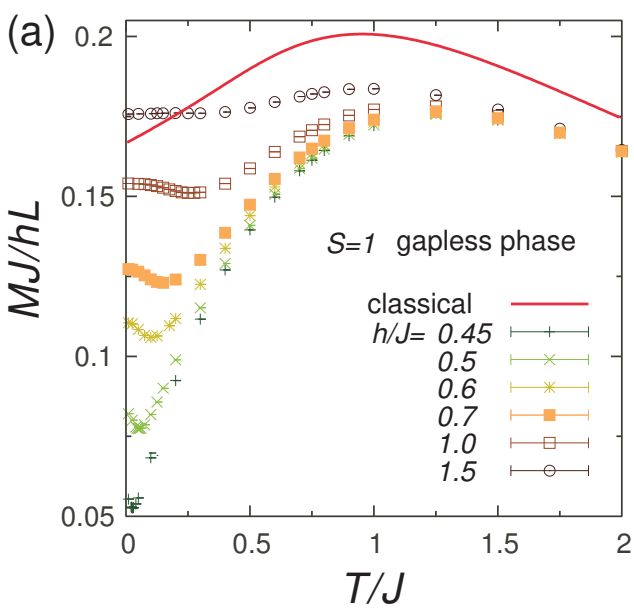

(b)

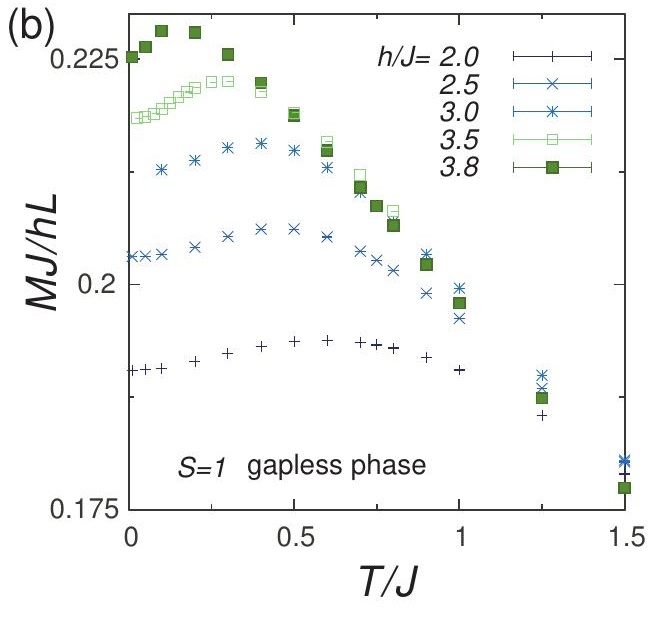



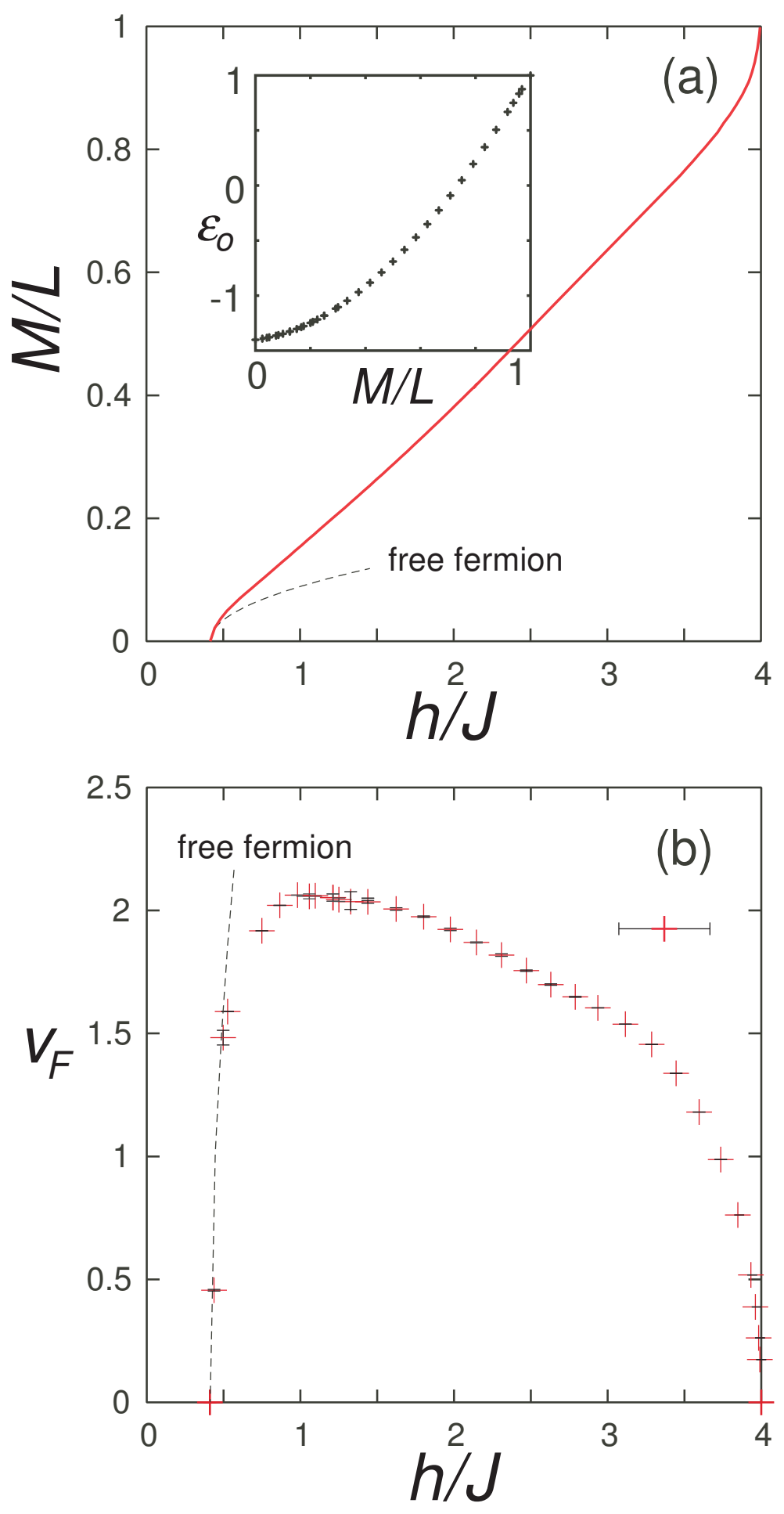